\title{
Twisted D3-brane and M5-brane compactifications from multi-charge spindles
}

\author{
Andrea Boido, Juan Manuel Pérez Ipiña and James Sparks \\ Mathematical Institute, University of Oxford, \\ Andrew Wiles Building, Radcliffe Observatory Quarter, \\ Woodstock Road, Oxford, OX2 6GG, U.K. \\ E-mail: andrea.boido@maths.ox.ac.uk, Juan.PerezIpina@maths.ox.ac.uk, \\ james.sparks@maths.ox.ac.uk
}

ABSTRACT: We construct families of supersymmetric $\mathrm{AdS}_{3} \times Y_{7}$ and $\mathrm{AdS}_{3} \times Y_{8}$ solutions to type IIB string theory and M-theory, respectively. Here $Y_{7}$ is an $S^{5}$ fibration over $\Sigma$, while $Y_{8}$ is an $S^{4}$ fibration over $\Sigma_{g} \times \Sigma$, where $\Sigma_{g}$ is a Riemann surface of genus $g>1$ and $\Sigma$ is a two-dimensional orbifold known as a spindle. We interpret the solutions as near-horizon limits of $N$ D3-branes wrapped on $\Sigma$ and $N$ M5-branes wrapped on $\Sigma_{g} \times \Sigma$, respectively. These are holographically dual to $d=2,(0,2)$ SCFTs, and we show that the central charge and superconformal R-symmetry of the gravity solutions agree with dual field theory calculations.

KeYwords: AdS-CFT Correspondence, D-branes, M-Theory, Supergravity Models

ArXiv EPrint: 2104.13287 


\section{Contents}

1 Introduction and summary 1

2 D3-brane supergravity solutions $\quad 4$

2.1 Local form of the solutions 4

$\begin{array}{ll}2.2 & \text { Global analysis and general solution }\end{array}$

2.3 Central charge and R-symmetry 8

$\begin{array}{lll}2.4 & \text { Special cases } & 10\end{array}$

3 M5-brane supergravity solutions 11

$\begin{array}{lll}3.1 & \text { Romans supergravity and uplift } & 11\end{array}$

$\begin{array}{ll}3.2 \text { Central charge } & 13\end{array}$

4 Field theory $\quad 14$

4.1 D3-brane anomaly polynomial 14

$\begin{array}{lll}4.2 & c \text {-extremization and superconformal R-symmetry } & 16\end{array}$

$\begin{array}{lll}4.3 & \text { M5-brane anomaly polynomial } & 16\end{array}$

5 Discussion 17

\section{Introduction and summary}

Interesting classes of superconformal field theories (SCFTs) may be obtained in string theory and M-theory by wrapping branes on a compact space $\Sigma$, and flowing to the infrared (IR). Supergravity solutions describing the near-horizon limits of such wrapped branes were first constructed in the pioneering work of [1], describing D3-branes and M5-branes wrapping a constant curvature Riemann surface $\Sigma_{g}$ of genus $g>1$. This work has subsequently been generalized in many directions, leading to a large literature on the subject.

Until recently such constructions have realized supersymmetry on the wrapped brane worldvolume via a so-called topological twist [2]. Here the spin connection on the wrapped directions $\Sigma$ is effectively cancelled by the coupling of the spinor to external R-symmetry gauge fields, which geometrically are connections on the normal bundle to $\Sigma$ in spacetime. The upshot is that the preserved Killing spinors are sections of trivial bundles over $\Sigma$, and in fact constant. A different realization of supersymmetry has recently been presented in [3]. Here a family of supersymmetric $\mathrm{AdS}_{3} \times \Sigma$ solutions was constructed in $d=5$ minimal gauged supergravity, where $\Sigma$ is a two-dimensional orbifold surface known as a spindle. This is topologically a two-sphere, but with conical deficit angles $2 \pi\left(1-\frac{1}{n_{+}}\right)$at the two poles, specified by coprime positive integers $n_{+} \neq n_{-}$. Equivalently, $\Sigma=\mathbb{W} \mathbb{C P}_{\left[n_{-}, n_{+}\right]}^{1}$ is a weighted projective space. When $n_{-}-n_{+}$is divisible by 3 these may be uplifted on 
an $S^{5}$ internal space, leading to $\mathrm{AdS}_{3} \times Y_{7}$ solutions of type IIB supergravity in which $Y_{7}$ takes the fibred form

$$
S^{5} \hookrightarrow Y_{7} \rightarrow \Sigma=\mathbb{W} \mathbb{C P}_{\left[n_{-}, n_{+}\right]}^{1} .
$$

Since these ten-dimensional solutions are sourced only by five-form flux $F_{5}$, with $N$ units of flux through the $S^{5}$ fibre of (1.1), these are naturally interpreted as near-horizon limits of $N$ D3-branes wrapped on the spindle $\Sigma$. However, the Killing spinors on $\Sigma$ are not constant, and moreover are sections of non-trivial spinor bundles [4]. The flux through $\Sigma$ of the $d=5$ Abelian R-symmetry gauge field in gauged supergravity is correspondingly not simply proportional to the Euler number $\chi(\Sigma)$, given by

$$
\chi(\Sigma)=\frac{n_{-}+n_{+}}{n_{-} n_{+}}
$$

as is characteristic of a topological twist.

In the first part of this paper we generalize the construction of [3] by allowing for more general fibrations in (1.1). More precisely, the $d=5$ minimal gauged supergravity solution of [3] is a special case of a more general class of (local) $\mathrm{AdS}_{3}$ solutions to $d=5$, $\mathrm{U}(1)^{3}$ gauged supergravity with two additional parameters, first constructed in [5]. These also uplift on $S^{5}$, with the three gauge fields of $\mathrm{U}(1)^{3}$ determining the fibration of this internal space over the $d=5$ spacetime. The resulting supersymmetric $\mathrm{AdS}_{3} \times Y_{7}$ solutions were in fact constructed before reference [5], in [6]. The approach we take to the global analysis of these supergravity solutions is different to that in $[5,6]$, and instead follows [3]. Specifically, we first construct $\mathrm{AdS}_{3} \times \Sigma$ solutions of the $d=5, \mathrm{U}(1)^{3}$ gauged supergravity theory, where $\Sigma=\mathbb{W} \mathbb{C P}_{\left[n_{-}, n_{+}\right]}^{1}$ is a spindle specified by coprime positive integers $n_{-}>n_{+}$. We then appropriately quantize the three Abelian R-symmetry gauge field fluxes through $\Sigma$, in such a way that the resulting fibration (1.1) is well-defined, with a smooth total space $Y_{7}$. More precisely, defining the fluxes

$$
Q_{I} \equiv \frac{1}{2 \pi} \int_{\Sigma} F^{(I)}
$$

where $F^{(I)}, I=1,2,3$, are the three Abelian R-symmetry gauge field curvatures, we construct solutions with

$$
Q_{1}=\frac{q}{n_{-} n_{+}}, \quad Q_{2}=\frac{p}{n_{-} n_{+}}, \quad Q_{3}=\frac{n_{-}-n_{+}-q-p}{n_{-} n_{+}} .
$$

Here $q, p>0$ are positive integers, constrained so that $q+p<n_{-}-n_{+}$, and where $q$ and $p$ have no common factor with either of $n_{-}, n_{+}$. Notice that the charges (1.4) satisfy

$$
Q_{1}+Q_{2}+Q_{3}=\frac{n_{-}-n_{+}}{n_{-} n_{+}} .
$$

This should be contrasted with a topological twist, where instead on the right hand side of (1.5) one would have the Euler number of the spindle (1.2). The solutions of [3] are recovered by setting $Q_{1}=Q_{2}=Q_{3}$, or equivalently $p=q=\frac{1}{3}\left(n_{-}-n_{+}\right)$, which then requires $n_{-}-n_{+}$to be divisible by 3 . 
The central charge of these solutions is given by

$$
c=\frac{3 p q\left(n_{-}-n_{+}-q-p\right)}{n_{-} n_{+}\left[\left(n_{-}-n_{+}\right)(p+q)+n_{-} n_{+}-p^{2}-p q-q^{2}\right]} N^{2} .
$$

The solution is thus specified by five positive integers: $n_{-}, n_{+}$determine the topology of the wrapped spindle $\Sigma=\mathbb{W} \mathbb{C P}_{\left[n_{-}, n_{+}\right]}^{1}, q, p$ determine the R-symmetry fluxes (1.4) and hence twisting in the fibration (1.1), and finally $N$ is the number of wrapped D3-branes. As in [3], the description of these solutions suggests that the dual $d=2,(0,2)$ SCFTs arise from a twisted compactification of $\mathcal{N}=4 \mathrm{SYM}$ on $\Sigma$, where there are background R-symmetry fluxes given by (1.4). We make a check on this conjecture by computing both the central charge and superconformal R-symmetry of the $d=2$ field theories, making use of the anomaly polynomial of $\mathcal{N}=4 \mathrm{SYM}$ together with $c$-extremization [7]. We find precise agreement with the corresponding supergravity quantities.

When $Q_{1}=Q_{2}$, or equivalently $p=q$, the solutions to $d=5, \mathrm{U}(1)^{3}$ supergravity are also solutions to Romans $\mathcal{N}=4, \mathrm{SU}(2) \times \mathrm{U}(1)$ supergravity [8]. The latter is a consistent truncation of $d=11$ supergravity on the Maldacena-Núñez solution referred to at the start of this introduction [9]. We thus also obtain $\mathrm{AdS}_{3} \times Y_{8}$ solutions of $d=11$ supergravity, where $Y_{8}$ takes the fibred form

$$
S^{4} \hookrightarrow Y_{8} \rightarrow \Sigma_{g} \times \Sigma .
$$

Here $\Sigma_{g}$ is a constant curvature Riemann surface of genus $g>1$. The original MaldacenaNúñez solution corresponds to the $\mathrm{AdS}_{5}$ vacuum of the Romans theory, and is dual to the $d=4, \mathcal{N}=2$ SCFT living on $N$ M5-branes wrapped on the Riemann surface $\Sigma_{g}$, which is holomorphically embedded inside a Calabi-Yau two-fold. The uplift to $d=11$ of our $Q_{1}=Q_{2}$ solution, with parameter $q$, then describes the further twisted compactification of this four-dimensional theory on the spindle $\Sigma$, with $0<q<\frac{1}{2}\left(n_{-}-n_{+}\right)$determining the twisting. The central charge of these gravity solutions is

$$
c=\frac{4 q^{2}\left(n_{-}-n_{+}-2 q\right)}{n_{-} n_{+}\left[n_{-}\left(n_{+}+2 q\right)-q\left(2 n_{+}+3 q\right)\right]}(g-1) N^{3} .
$$

We again reproduce this result from a dual field theory calculation, this time utilizing the anomaly polynomial of the theory on $N$ M5-branes, doubly wrapped on $\Sigma_{g} \times \Sigma$.

The outline of the paper is as follows. In section 2 we construct the $\operatorname{AdS}_{3} \times Y_{7}$ solutions of interest by first constructing $d=5 \mathrm{AdS}_{3} \times \Sigma$ solutions in $\mathrm{U}(1)^{3}$ gauged supergravity, and then imposing appropriate quantization conditions on the gauge field fluxes through the spindle $\Sigma=\mathbb{W} \mathbb{C P}_{\left[n_{-}, n_{+}\right]}^{1}$ so that the uplift on $S^{5}$ leads to a smooth seven-manifold $Y_{7}$. We also compute the central charge and R-symmetry gauge field for these gravity solutions. In section 3 we similarly construct $\mathrm{AdS}_{3} \times Y_{8}$ solutions to M-theory by uplifting the same $d=5$ solutions, but now necessarily with $Q_{1}=Q_{2}$, and compute the central charge. In section 4 we compute the $d=2$ anomaly polynomial for the twisted compactification of $\mathcal{N}=4 \mathrm{SYM}$ on $\Sigma$, and using $c$-extremization then compute the exact central charge and superconformal R-symmetry in field theory, finding agreement with the gravity duals. We perform a similar computation for the theory on $N$ M5-branes wrapped on $\Sigma_{g} \times \Sigma$, again 
finding agreement with the central charge computed from the supergravity solution. We conclude in section 5 with a brief discussion.

Note added. As this paper was being completed we learned of the work [10], which has overlap with the D3-brane results presented here.

\section{D3-brane supergravity solutions}

In this section we construct a family of supersymmetric $\mathrm{AdS}_{3}$ solutions to $d=5, \mathrm{U}(1)^{3}$ gauged supergravity. These uplift on an $S^{5}$ internal space to corresponding $\operatorname{AdS}_{3} \times Y_{7}$ solutions of type IIB supergravity. The local form of the solutions was first presented in [6], where they were obtained from multi-charge superstar solutions in [11]. Shortly afterwards the same solutions were rediscovered in [5], where they arise as the uplifts of near-horizon limits of a family $d=5$ unbalanced black ring solutions. The novelty of this section is the global analysis that we perform for the allowed values of the parameters in the solution, which is very different to the approach taken in [5] and [6]. Instead we follow [3]. The resulting description will be crucial to match the dual field theory computation in section 4 .

\subsection{Local form of the solutions}

The action of $d=5, \mathrm{U}(1)^{3}$ gauged supergravity is

$$
\begin{aligned}
S=\frac{1}{16 \pi G_{(5)}} \int\left\{\mathrm{d}^{5} x \sqrt{-\operatorname{det} g}[\right. & R+4 \sum_{I=1}^{3}\left(X^{(I)}\right)^{-1}-\frac{1}{2} \sum_{I=1}^{3}\left(X^{(I)}\right)^{-2}\left(\partial X^{(I)}\right)^{2} \\
& \left.\left.-\frac{1}{4} \sum_{I=1}^{3}\left(X^{(I)}\right)^{-2}\left(F^{(I)}\right)^{2}\right]-F^{(1)} \wedge F^{(2)} \wedge A^{(3)}\right\} .
\end{aligned}
$$

Here $A^{(I)}, I=1,2,3$, are the three $\mathrm{U}(1)$ gauge fields, with field strengths $F^{(I)}=\mathrm{d} A^{(I)}$, and the three scalar fields $X^{(I)}$ satisfy the constraint $X^{(1)} X^{(2)} X^{(3)}=1$. We have set the gauge coupling constant to 1 . A consistent truncation to minimal gauged supergravity may be obtained by setting the scalars $X^{(I)}=1$ and the three gauge fields equal $A^{(I)}=A$, $I=1,2,3$, where to compare to the conventions in [3] one should also rescale the gauge field $A$ by factor of $\frac{3}{2}$.

Our starting point is the following supersymmetric $\mathrm{AdS}_{3}$ solution to this theory [5]:

$$
\begin{aligned}
\mathrm{d} s_{5}^{2} & =H(x)^{1 / 3}\left[\mathrm{~d} s_{\mathrm{AdS}_{3}}^{2}+\frac{1}{4 P(x)} \mathrm{d} x^{2}+\frac{P(x)}{H(x)} \mathrm{d} \phi^{2}\right], \\
A^{(I)} & =\frac{x-\alpha}{x+3 K_{I}} \mathrm{~d} \phi \\
X^{(I)} & =\frac{H(x)^{1 / 3}}{x+3 K_{I}} .
\end{aligned}
$$


Here $\mathrm{d} s_{\mathrm{AdS}_{3}}^{2}$ a unit radius metric on $\mathrm{AdS}_{3}$, the metric functions are the polynomials

$$
\begin{aligned}
H(x) & \equiv\left(x+3 K_{1}\right)\left(x+3 K_{2}\right)\left(x+3 K_{3}\right)=x^{3}+3 c_{1} x+c_{2}, \\
P(x) & \equiv H(x)-(x-\alpha)^{2},
\end{aligned}
$$

and $\alpha, K_{I}$ are constants, with the latter satisfying the constraint $K_{1}+K_{2}+K_{3}=0$. In the first line of (2.3) we have introduced the elementary symmetric polynomials for the parameters $K_{I}$, namely $c_{1} \equiv 3\left(K_{1} K_{2}+K_{2} K_{3}+K_{1} K_{3}\right), c_{2} \equiv 27 K_{1} K_{2} K_{3}$. We note that the solution to minimal gauged supergravity studied in [3] is obtained by setting $K_{I}=0$ for all $I=1,2,3$, which is then parametrized by the single parameter $\alpha$.

Any solution of $d=5, \mathrm{U}(1)^{3}$ gauged supergravity may be uplifted (locally) on an $S^{5}$ internal space to a solution of type IIB supergravity [11]. The ten-dimensional metric is

$$
L^{-2} \mathrm{~d} s_{10}^{2}=W^{1 / 2} \mathrm{~d} s_{5}^{2}+W^{-1 / 2} \sum_{I=1}^{3}\left(X^{I}\right)^{-1}\left[\mathrm{~d} \mu_{I}^{2}+\mu_{I}^{2}\left(\mathrm{~d} \phi_{I}+A^{(I)}\right)^{2}\right],
$$

while the self-dual five-form flux is

$$
\begin{aligned}
L^{-4} F_{5}=\left(1+\star_{10}\right)\{ & 2 \sum_{I=1}^{3}\left[\left(X^{(I)}\right)^{2} \mu_{I}^{2}-W X^{(I)}\right] \operatorname{vol}_{5}+\frac{1}{2} \sum_{I=1}^{3}\left(X^{(I)}\right)^{-1} \star_{5} \mathrm{~d} X^{(I)} \wedge \mathrm{d}\left(\mu_{I}^{2}\right) \\
& \left.+\frac{1}{2} \sum_{I=1}^{3}\left(X^{(I)}\right)^{-2} \mathrm{~d}\left(\mu_{I}^{2}\right) \wedge\left(\mathrm{d} \phi_{I}+A^{(I)}\right) \wedge \star_{5} F^{(I)}\right\} .
\end{aligned}
$$

Here we have introduced an arbitrary length scale parameter $L>0$ into the form of the solution, which will be fixed via flux quantization later. We have denoted the volume form on $\mathrm{d} s_{5}^{2}$ by $\mathrm{vol}_{5}$, and $\star$ denotes the Hodge duality operator in the appropriate dimension. As in [6] we have corrected the sign of the second factor in (2.5), compared with the expression given in [11]. We have also introduced the warp factor function

$$
W=\sum_{I=1}^{3} \mu_{I}^{2} X^{(I)}>0 .
$$

Finally, $\left\{\mu_{I}, \phi_{I}\right\}$ form a system of polar coordinates on $S^{5} \subset \mathbb{R}^{2} \oplus \mathbb{R}^{2} \oplus \mathbb{R}^{2}$, where correspondingly $\sum_{I=1}^{3} \mu_{I}^{2}=1$ and the angular coordinates $\phi_{I}$ each have period $2 \pi$. Notice that the warp factor function (2.6) depends on both the internal $S^{5}$ coordinates $\mu_{I}$ and a priori on the $d=5$ coordinates, via the scalar fields $X^{(I)}$.

Applying the uplifting formula (2.4) to the $\mathrm{AdS}_{3}$ solution (2.2) gives rise to the following warped $\mathrm{AdS}_{3} \times Y_{7}$ metric

$$
\mathrm{d} s_{10}^{2}=L^{2} W^{1 / 2} H(x)^{1 / 3}\left(\mathrm{~d} s_{\mathrm{AdS}_{3}}^{2}+\mathrm{d} s_{Y_{7}}^{2}\right),
$$

where the seven-dimensional metric on $Y_{7}$ is

$$
\begin{aligned}
\mathrm{d} s_{Y_{7}}^{2}= & \frac{\mathrm{d} x^{2}}{4 P(x)}+\frac{P(x)}{H(x)} \mathrm{d} \phi^{2} \\
& +\frac{1}{W H(x)^{2 / 3}} \sum_{I=1}^{3}\left(x+3 K_{I}\right)\left[\mathrm{d} \mu_{I}^{2}+\mu_{I}^{2}\left(\mathrm{~d} \phi_{I}+\frac{(x-\alpha)}{x+3 K_{I}} \mathrm{~d} \phi\right)^{2}\right] .
\end{aligned}
$$


One can verify that this is the same metric as that given in section 5.2 of [6], where we identify their coordinates and parameters, in terms of those presented here, as $w=x-\alpha$, $z=-2 \phi, q_{I}=\alpha+3 K_{I}$. In particular, reference [6] also shows that the solution is supersymmetric, with the dual $d=2$ CFT having $(0,2)$ supersymmetry.

\subsection{Global analysis and general solution}

Given a local $\mathrm{AdS}_{3} \times Y_{7}$ solution of the form (2.8), one would like to choose the parameters $\alpha, K_{I}$ and ranges of coordinates so as to obtain a smooth metric on a compact internal space $Y_{7}$. An analysis of this was carried out in [6] (see also [5]), where one looks at the degeneration loci of Killing vector fields on $Y_{7}$, which are constant linear combinations of the four vector fields $\partial_{\phi}$ and $\partial_{\phi_{I}}, I=1,2,3$. We instead follow the approach of [3], first obtaining a suitably regular $d=5 \mathrm{AdS}_{3} \times \Sigma$ solution, where $\Sigma=\mathbb{W C P}_{\left[n_{-}, n_{+}\right]}^{1}$ is a twodimensional orbifold known as a spindle. We then appropriately quantize the $\mathrm{U}(1)^{3}$ gauge field fluxes so as to obtain a regular fibration of $S^{5}$ over this five-dimensional solution.

We begin then with the $d=5$ metric in (2.2), which we rewrite as

$$
\mathrm{d} s_{5}^{2}=H(x)^{1 / 3}\left(\mathrm{~d} s_{\mathrm{AdS}_{3}}^{2}+\mathrm{d} s_{\Sigma}^{2}\right),
$$

with the two-dimensional surface $\Sigma$ having coordinates $x, \phi$. In order for the metric to have the correct signature and be non-singular we would like to choose the parameters so that $H(x)>0$ is strictly positive, and similarly $P(x) \geq 0$. In order that $\Sigma$ forms a compact surface without boundary, we furthermore assume that $x \in\left[x_{1}, x_{2}\right]$ lies between two roots of the polynomial $P(x)$ in (2.3). In a certain regime of parameters, that we make explicit below, we find that the three roots $x_{i}, i=1,2,3$, of $P(x)$ are real and positive, and we then take $0<x_{1}<x_{2}<x_{3}$ so that $P(x) \geq 0$ for $x \in\left[x_{1}, x_{2}\right]$. Defining $\varrho_{i}=2\left(x-x_{i}\right)^{1 / 2}$ for $i=1,2$ we find that as $x \rightarrow x_{i}$ the metric on the two-dimensional surface $\Sigma$ approaches

$$
\mathrm{d} s_{\Sigma}^{2} \simeq \frac{1}{4 P^{\prime}\left(x_{i}\right)}\left(\mathrm{d} \varrho_{i}^{2}+\kappa_{i}^{2} \varrho_{i}^{2} \mathrm{~d} \phi^{2}\right), \quad \text { where } \quad \kappa_{i} \equiv\left|\frac{P^{\prime}\left(x_{i}\right)}{x_{i}-\alpha}\right|, \quad i=1,2 .
$$

As for the solution in [3], it is not possible to remove both conical singularities at the roots $x=x_{i}$ by making a single choice $\Delta \phi$ of the period for $\phi$. Instead we impose

$$
\Delta \phi=\frac{2 \pi}{\kappa_{1} n_{+}}=\frac{2 \pi}{\kappa_{2} n_{-}},
$$

where $n_{ \pm} \in \mathbb{N}$. The resulting surface $\Sigma=\mathbb{W} \mathbb{C P}_{\left[n_{-}, n_{+}\right]}^{1}$ is then an orbifold known as a spindle. This is topologically a two-sphere, but with conical deficit angles $2 \pi\left(1-\frac{1}{n_{ \pm}}\right)$at the poles $x=x_{1}, x_{2}$.

After a little computation we find that the second equality in (2.11) may be written as

$$
\frac{n_{-}}{n_{+}}=\left|\frac{x_{2}-\alpha}{x_{1}-\alpha}\right|\left|\frac{-1+2 x_{1}+x_{2}}{-1+x_{1}+2 x_{2}}\right| \text {, }
$$

where the roots $x_{1}, x_{2}$ satisfy the equations

$$
\begin{aligned}
2 \alpha+3 c_{1} & =x_{1}+x_{2}-x_{1}^{2}-x_{2}^{2}-x_{1} x_{2}, \\
c_{2} & =\alpha^{2}+x_{1}^{2} x_{2}+x_{1} x_{2}^{2}-x_{1} x_{2},
\end{aligned}
$$

and we have eliminated $x_{3}$ via the relation $x_{1}+x_{2}+x_{3}=1$. 
We will also need the fluxes of the gauge field strengths $F^{(I)}=\mathrm{d} A^{(I)}$ through the surface $\Sigma$. Using the expression for $A^{(I)}$ in $(2.2)$ we compute

$$
Q_{I} \equiv \frac{1}{2 \pi} \int_{\Sigma} F^{(I)}=\frac{\left(x_{2}-x_{1}\right)\left(\alpha+3 K_{I}\right)}{\left(x_{1}+3 K_{I}\right)\left(x_{2}+3 K_{I}\right)} \frac{\Delta \phi}{2 \pi} .
$$

As explained in $[3,4]$, for $\Sigma=\mathbb{W} \mathbb{C P}_{\left[n_{-}, n_{+}\right]}^{1}$ the appropriate quantization condition on these fluxes is that

$$
Q_{I} \equiv \frac{p_{I}}{n_{-} n_{+}}, \quad \text { where } \quad p_{I} \in \mathbb{Z} .
$$

Specifically, the circles parametrized by $\phi_{I}$ inside the internal space $S^{5} \subset \mathbb{C}^{3}$ then give a well-defined orbifold circle fibration over $\Sigma$. Moreover, provided the integers $p_{I}$ are coprime to both of $n_{ \pm}$, the total space of this fibration is smooth. ${ }^{1}$ This leads to a fibration

$$
S^{5} \hookrightarrow Y_{7} \rightarrow \Sigma=\mathbb{W} \mathbb{C P}\left[n_{\left.-, n_{+}\right]},\right.
$$

where the total space $Y_{7}$ is a compact seven-manifold, and the twisting is determined by the integers $p_{I}$.

At this stage we have a three-parameter family of solutions, determined by the constants $\alpha, K_{1}, K_{2}$, where the roots $x_{1}, x_{2}$ obey (2.13). We would like to express the solution in terms of the more physical flux parameters $p_{I}$ and $n_{ \pm}$, the ratio of the latter determined via (2.12). It turns out to be convenient to express quantities in terms of two of the three fluxes, which we take to be

$$
p_{1} \equiv q, \quad p_{2} \equiv p .
$$

After some work, it is possible to solve the three equations (2.12), and (2.13) for $\alpha, K_{1}, K_{2}$, $x_{1}$, and $x_{2}$ in terms of $n_{ \pm}$, together with $q$ and $p$. We find the rather unwieldy expressions

$$
\begin{aligned}
x_{1}= & \frac{1}{\mathcal{D}}\left\{\left(p^{2}+p q+q^{2}\right)^{2}+3 n_{+} p q\left(p+q+n_{+}\right)+2\left(n_{+}-n_{-}\right)(p+q)\left(p^{2}+q^{2}\right)\right. \\
& \left.+\left(n_{-}^{2}+n_{+}^{2}\right)\left(p^{2}+q^{2}\right)-n_{-} n_{+}\left(3 p^{2}+4 p q+3 q^{2}\right)+n_{-} n_{+}\left(n_{-}-n_{+}\right)(p+q)\right\}, \\
x_{2}= & \frac{1}{\mathcal{D}}\left\{\left(p^{2}+p q+q^{2}\right)^{2}-3 n_{-} p q\left(p+q-n_{-}\right)+2\left(n_{+}-n_{-}\right)(p+q)\left(p^{2}+q^{2}\right)\right. \\
& \left.+\left(n_{-}^{2}+n_{+}^{2}\right)\left(p^{2}+q^{2}\right)-n_{-} n_{+}\left(3 p^{2}+4 p q+3 q^{2}\right)+n_{-} n_{+}\left(n_{-}-n_{+}\right)(p+q)\right\}, \\
K_{1}= & \frac{1}{3 \mathcal{D}}\left\{3 p^{2}(p+q)^{2}-\left(p^{2}+p q+q^{2}\right)^{2}+2\left(n_{-}-n_{+}\right)\left(q^{2}-2 p^{2}\right)(p+q)-2 n_{-} n_{+} p q\right. \\
& \left.-\left(n_{-}^{2}+n_{+}^{2}\right)\left(q^{2}-2 p^{2}\right)+3 n_{-} n_{+}\left(q^{2}-2 p^{2}\right)-n_{-} n_{+}\left(n_{-}-n_{+}\right)(q-2 p)\right\}, \\
K_{2}= & \frac{1}{3 \mathcal{D}}\left\{3 q^{2}(p+q)^{2}-\left(p^{2}+p q+q^{2}\right)^{2}+2\left(n_{-}-n_{+}\right)\left(p^{2}-2 q^{2}\right)(p+q)-2 n_{-} n_{+} p q\right. \\
& \left.-\left(n_{-}^{2}+n_{+}^{2}\right)\left(p^{2}-2 q^{2}\right)+3 n_{-} n_{+}\left(p^{2}-2 q^{2}\right)-n_{-} n_{+}\left(n_{-}-n_{+}\right)(p-2 q)\right\},
\end{aligned}
$$

\footnotetext{
${ }^{1}$ See appendix $\mathrm{A}$ of [4] for a detailed discussion of this.
} 
where we have defined the denominator term

$$
\mathcal{D} \equiv 3\left[\left(n_{-}-n_{+}\right)(p+q)+n_{-} n_{+}-p^{2}-p q-q^{2}\right]^{2} .
$$

The parameter $\alpha$ may then be determined from the first equation in (2.13).

The third flux is computed to be

$$
Q_{3}=\frac{n_{-}-n_{+}-q-p}{n_{-} n_{+}},
$$

which notice also satisfies the quantization condition (2.15), with

$$
p_{1}=q, \quad p_{2}=p, \quad p_{3}=n_{-}-n_{+}-q-p .
$$

We then also have

$$
Q_{1}+Q_{2}+Q_{3}=\frac{n_{-}-n_{+}}{n_{-} n_{+}} .
$$

We find that the roots obey the assumed inequalities $0<x_{1}<x_{2}<x_{3}$ provided

$$
q, p>0, \quad q+p<n_{-}-n_{+} .
$$

These inequalities also ensure that the denominator (2.19) is non-vanishing and hence $\mathcal{D}>0$.

We now have a family of regular supersymmetric $\mathrm{AdS}_{3} \times Y_{7}$ solutions, with $Y_{7}$ having the fibration structure (2.16), parametrized by the integers $n_{-}, n_{+}, p$ and $q$. We address quantization of the five-form flux $F_{5}$ in the next subsection.

\subsection{Central charge and R-symmetry}

The $\mathrm{AdS}_{3}$ solutions are, via the AdS/CFT correspondence, expected to be dual to $d=2$, $(0,2)$ SCFTs. The central charge of these CFTs is computed in gravity using the standard formula [12]

$$
c=\frac{3 L}{2 G_{(3)}},
$$

where $G_{(3)}$ is the effective Newton constant in three dimensions. In turn the latter is computed via dimensional reduction on $Y_{7}$, and is given by [13]

$$
\frac{1}{G_{(3)}}=\frac{L^{7}}{G_{(10)}} \int_{Y_{7}} W^{2} H(x)^{4 / 3} \operatorname{vol}_{7}=\frac{\pi^{3} L^{7} \Delta \phi}{2 G_{(10)}}\left(x_{2}-x_{1}\right),
$$

where we have explicitly evaluated the integrals over $S^{5}$ and the spindle $\Sigma$. The tendimensional Newton constant is

$$
G_{(10)}=\frac{(2 \pi)^{7} g_{s}^{2} \ell_{s}^{8}}{16 \pi},
$$

where $\ell_{s}$ and $g_{s}$ are the constant string length and string coupling constant, respectively. The central charge (2.24) is then

$$
c=\frac{3 L^{8} \Delta \phi}{32 \pi^{3} g_{s}^{2} \ell_{s}^{8}}\left(x_{2}-x_{1}\right) .
$$


In order to obtain a good string theory solution we must also quantize the flux of the closed five-form (2.5) through five-cycles in the ten-dimensional spacetime. In particular, integrating $F_{5}$ through a copy of the $S^{5}$ fibre, at any point in the $d=5$ spacetime, we find the total flux is

$$
N \equiv \frac{1}{\left(2 \pi \ell_{s}\right)^{4} g_{s}} \int_{S^{5}} F_{5}=\frac{L^{4}}{4 \pi \ell_{s}^{4} g_{s}} .
$$

Eliminating $L$ in the central charge (2.27) using this last equation then gives

$$
c=\frac{3}{2 \pi} \Delta \phi\left(x_{2}-x_{1}\right) N^{2} .
$$

Finally, substituting the solution (2.18) into (2.29), we find the remarkably simple expression

$$
c=\frac{3 p q\left(n_{-}-n_{+}-q-p\right)}{n_{-} n_{+}\left[\left(n_{-}-n_{+}\right)(p+q)+n_{-} n_{+}-p^{2}-p q-q^{2}\right]} N^{2} .
$$

This is our final formula for the central charge, parametrized in terms of the integers $n_{-}, n_{+}, p, q$ and $N$. It will be useful to note that (2.11) gives the period $\Delta \phi$ of $\phi$ to be

$$
\frac{\Delta \phi}{2 \pi}=\frac{\left(n_{-}-n_{+}\right)(p+q)+n_{-} n_{+}-p^{2}-p q-q^{2}}{n_{-} n_{+}\left(n_{-}+n_{+}\right)},
$$

an expression we will need momentarily.

The U(1) R-symmetry of the dual $d=2,(0,2)$ field theory is realized in the gravity solution as a Killing vector field $R_{2 \mathrm{~d}}$ of the $\mathrm{AdS}_{3} \times Y_{7}$ gravity solution. This is defined as a certain bilinear in the Killing spinors on $Y_{7}$ [14], and combining the general results of the latter reference with the form of the solution given in [6] we can read off that this vector field is simply

$$
R_{2 \mathrm{~d}}=\partial_{\phi}
$$

Here we have normalized the R-symmetry so that the Killing spinor on $Y_{7}$ has unit charge under the Lie derivative along this vector field, giving an explicit phase dependence of $\mathrm{e}^{\mathrm{i} \phi}$. On the other hand, this Killing spinor arises as a tensor product of the $d=5$ Killing spinor with a spinor on the internal $S^{5}$. This ansatz preserves an $\mathcal{N}=1$ spinor, out of the full $\mathcal{N}=4$ supersymmetry of $S^{5}$, with the spinor on $S^{5}$ having charge $\frac{1}{2}$ under each of the three vector fields $\partial_{\phi_{I}}$ generating the $\mathrm{U}(1)^{3}$ isometry of $S^{5} \subset \mathbb{C}^{3}$. As discussed in [3], we would like to choose a gauge for the $d=5$ gauge fields such that the Killing spinor is uncharged under the $\mathrm{U}(1)$ isometry that rotates the spindle. Since a gauge transformation of the $A^{(I)}$ precisely leads to a phase rotation in the corresponding Killing spinor, which has unit charge under each $A^{(I)}$, this may be achieved via the gauge/coordinate transformation

$$
\tilde{\phi}_{I}=\phi_{I}+\frac{2}{3} \phi, \quad \tilde{A}^{(I)}=A^{(I)}-\frac{2}{3} \mathrm{~d} \phi,
$$

which ensures that $\mathrm{d} \phi_{I}+A^{(I)}=\mathrm{d} \tilde{\phi}_{I}+\tilde{A}^{(I)}$. Defining also

$$
\varphi \equiv \frac{2 \pi}{\Delta \phi} \phi
$$


so that $\Delta \varphi=2 \pi$, and completing the coordinate transformation (2.33) by defining

$$
\tilde{\varphi}=\varphi
$$

we see that the R-symmetry vector $(2.32)$ is

$$
R_{2 \mathrm{~d}}=\partial_{\phi}=\frac{2 \pi}{\Delta \phi} \partial_{\varphi}=\frac{2 \pi}{\Delta \phi}\left(\sum_{I=1}^{3} \frac{\partial \tilde{\phi}_{I}}{\partial \varphi} \partial_{\tilde{\phi}_{I}}+\frac{\partial \tilde{\varphi}}{\partial \varphi} \partial_{\tilde{\varphi}}\right)=\frac{2}{3} \sum_{I=1}^{3} \partial_{\tilde{\phi}_{I}}+\frac{2 \pi}{\Delta \phi} \partial_{\tilde{\varphi}}
$$

Note that $\partial_{\tilde{\varphi}}$ generates the $\mathrm{U}(1)$ isometry of the spindle, that we refer to as $U(1)_{\mathcal{J}}$ in the subsequent discussion. The gauge transformation (2.33) leads to a phase $\mathrm{e}^{\mathrm{i}\left(\tilde{\phi}_{1}+\tilde{\phi}_{2}+\tilde{\phi}_{3}\right) / 2}$ in the Killing spinor on $Y_{7}$, but this is now invariant under $U(1)_{\mathcal{J}}$. We may then identify the Killing vector field on $S^{5}$

$$
R_{4 \mathrm{~d}}=\frac{2}{3} \sum_{I=1}^{3} \partial_{\tilde{\phi}_{I}}
$$

with the superconformal $\mathcal{N}=1$ R-symmetry before compactification of the theory on $\Sigma$, and from (2.36) hence write

$$
R_{2 \mathrm{~d}}=R_{4 \mathrm{~d}}+\frac{n_{-} n_{+}\left(n_{-}+n_{+}\right)}{\left(n_{-}-n_{+}\right)(p+q)+n_{-} n_{+}-p^{2}-p q-q^{2}} \partial_{\tilde{\varphi}} .
$$

Here in the second term we have substituted for $\Delta \phi$ using (2.31). Equation (2.38) states that the $d=4 \mathrm{U}(1)$ R-symmetry mixes with $U(1)_{\mathcal{J}}$ in flowing to the $d=2 \mathrm{U}(1) \mathrm{R}$ symmetry in the IR. We shall recover this formula from a dual field theory calculation in section 4 , along with the central charge (2.30).

\subsection{Special cases}

In this section we briefly analyse some interesting special cases of the general solutions of section 2.2, in particular making contact with [3].

Setting $p=q$ implies that the fluxes $Q_{1}=Q_{2}$ are equal, and hence also $A^{(1)}=A^{(2)}$, and $X^{(1)}=X^{(2)}$. In this case (2.18) simplifies to

$$
\begin{aligned}
& x_{1}=\frac{q\left(n_{+}+q\right)\left[2 n_{-}^{2}-2 n_{-}\left(n_{+}+4 q\right)+q\left(5 n_{+}+9 q\right)\right]}{3\left[n_{-}\left(n_{+}+2 q\right)-q\left(2 n_{+}+3 q\right)\right]^{2}}, \\
& x_{2}=-\frac{q\left(n_{-}-q\right)\left[2 n_{+}^{2}-2 n_{+}\left(n_{-}-4 q\right)-q\left(5 n_{-}-9 q\right)\right]}{3\left[n_{-}\left(n_{+}+2 q\right)-q\left(2 n_{+}+3 q\right)\right]^{2}}, \\
& K_{1}=K_{2}=\frac{q\left(n_{-}-n_{+}-3 q\right)\left(n_{+}+q\right)\left(n_{-}-q\right)}{9\left[n_{-}\left(n_{+}+2 q\right)-q\left(2 n_{+}+3 q\right)\right]^{2}},
\end{aligned}
$$

with corresponding central charge

$$
c=\frac{3 q^{2}\left(n_{-}-n_{+}-2 q\right)}{n_{-} n_{+}\left[n_{-}\left(n_{+}+2 q\right)-q\left(2 n_{+}+3 q\right)\right]} N^{2} .
$$

This sub-family of solutions also uplift to solutions of $d=11$ supergravity, as discussed in section 3 . 
Finally, setting all three charges equal gives, from (2.22),

$$
p=q=\frac{1}{3}\left(n_{-}-n_{+}\right) .
$$

This is precisely the solution to minimal gauged supergravity studied in [3], where we note that $p, q \in \mathbb{Z}$ then requires $n_{-}-n_{+}$to be divisible by 3 , as discussed in [3]. ${ }^{2}$ Imposing (2.41), our central charge (2.30) and R-symmetry gauge field (2.38) reduce to the expressions given in $[3]$.

\section{$3 \quad$ M5-brane supergravity solutions}

In this section we construct a family of supersymmetric $\mathrm{AdS}_{3} \times Y_{8}$ solutions to $d=11$ supergravity. These arise by uplifting the $Q_{1}=Q_{2}$ solutions of $d=5, \mathrm{U}(1)^{3}$ gauged supergravity in section 2.4 on the Maldacena-Núñez solution [1], using the consistent truncation of [9]. We interpret these M-theory solutions as the near-horizon limit of $N$ M5-branes wrapped on $\Sigma_{g} \times \Sigma$.

\subsection{Romans supergravity and uplift}

There are a number of different consistent truncations of type IIB supergravity on $S^{5}$, among them being both the $\mathrm{U}(1)^{3}$ gauged supergravity of section 2.1 , but also the Romans $\mathcal{N}=4, \mathrm{SU}(2) \times \mathrm{U}(1)$ supergravity [8]. The latter preserves a different subgroup of the full $\mathrm{SO}(6) \mathrm{R}$-symmetry of the internal $S^{5}$. The bosonic sector of the Romans theory contains a scalar field $X$, a triplet of $\mathrm{SU}(2)$ gauge fields $B_{\mu}^{i}, i=1,2,3$, a $\mathrm{U}(1)$ gauge field $A_{\mu}$, and two two-forms. This theory can be further truncated by setting to zero the two-forms and truncating $\mathrm{SU}(2)$ to its Abelian subgroup. It was shown in [15] that the resulting theory is the same as the $\mathrm{U}(1)^{3}$ theory with two gauge fields $A^{(1)}=A^{(2)}$ and two scalar fields $X^{(1)}=X^{(2)}$ set equal, and in particular then

$$
X=X^{(1)}, \quad B_{\mu}^{1}=B_{\mu}^{2}=0, \quad B_{\mu}^{3}=A_{\mu}^{(1)}, \quad A_{\mu}=A_{\mu}^{(3)} .
$$

On the other hand, this Romans supergravity theory is also a consistent truncation of $d=11$ supergravity, as shown in [9]. The vacuum $\mathrm{AdS}_{5}$ solution uplifts to a warped $\operatorname{AdS}_{5} \times N_{6}$ solution, which for a given internal space $N_{6}$ is dual to an $\mathcal{N}=2$ SCFT in four dimensions. A particular example, studied in [9], is the Maldacena-Núñez solution, which is dual to the $d=4, \mathcal{N}=2$ SCFT living on M5-branes wrapping a Riemann surface, holomorphically embedded in a Calabi-Yau two-fold. More generally there are the $\operatorname{AdS}_{5} \times N_{6}$ solutions of [16], corresponding to M5-branes wrapping a Riemann surface with punctures, and holographically dual to $d=4, \mathcal{N}=2$ SCFTs of class $\mathcal{S}$. Our five-dimensional solutions with $Q_{1}=Q_{2}$, given by (2.39), may thus also be uplifted to interesting classes of M5-brane solutions. Specifically, the M5-brane is wrapped on a Riemann surface (in general with

\footnotetext{
${ }^{2}$ In the notation of that reference, we take the Kähler-Einstein four-manifold to be $\mathrm{KE}_{4}=\mathbb{C P}^{2}$, and $k=1$ then gives $S^{5}$ as the internal space. But this then requires $n_{-}-n_{+}$to be divisible by the Fano index of $\mathbb{C P}^{2}$, which is $I=3$. The parameters $p$ and $q$ in this paper should not be confused with those in [3], and also recall that one should rescale our gauge field by a factor of $\frac{3}{2}$ to match to the conventions in [3].
} 
punctures), which is then further wrapped on the spindle $\Sigma=\mathbb{W} \mathbb{C P}_{\left[n_{-}, n_{+}\right]}^{1}$ to obtain a two-dimensional theory.

In this section for simplicity we focus on the uplift on the Maldacena-Núñez solution. A solution to the $d=5$ Romans theory with scalar field $X$, and Abelian gauge fields $B^{3}=A^{(1)}, A=A^{(3)}$, with $B^{1}=B^{2}=0$ as in (3.1), uplifts to a $d=11$ solution with metric $[9]^{3}$

$$
\begin{aligned}
L^{-2} \mathrm{~d} s_{11}^{2}= & 2^{-2 / 3} \Omega^{1 / 3} \mathrm{~d} s_{5}^{2}+2^{1 / 3} X \Omega^{1 / 3}\left(\mathrm{~d} \theta^{2}+\mathrm{d} s_{\Sigma_{g}}^{2}\right) \\
& +2^{1 / 3} X \Omega^{-2 / 3} \sin ^{2} \theta\left(\mathrm{d} \psi+V+A^{(3)}\right)^{2}+\frac{2^{-2 / 3}}{X^{2}} \Omega^{-2 / 3} \cos ^{2} \theta D \mu_{i} D \mu_{i} .
\end{aligned}
$$

Here $\mathrm{d} s_{5}^{2}$ denotes the five-dimensional gauged supergravity metric, $\mathrm{d} s_{\Sigma_{g}}^{2}$ is the metric on a unit radius hyperbolic plane, quotiented by a discrete group of isometries to obtain a compact Riemann surface of genus $g>1$, and we have introduced the warp factor function

$$
\Omega \equiv \cos ^{2} \theta+\frac{1}{2 X^{3}} \sin ^{2} \theta .
$$

The coordinates $\theta, \psi$, and $\mu_{i}, i=1,2,3$, are (constrained) coordinates on $S^{4} \subset \mathbb{R}^{2} \oplus \mathbb{R}^{3}$, where $\theta \in\left[0, \frac{\pi}{2}\right]$ describes the polar direction in the latter splitting. Smoothness of the metric (3.2) at $\theta=0$ fixes the period $\Delta \psi=2 \pi$, and the local one-form $V$ on $\Sigma_{g}$ is such that $\mathrm{d} V=-\operatorname{vol}_{\Sigma_{g}}$. We note that

$$
\operatorname{Vol}\left(\Sigma_{g}\right)=\int_{\Sigma_{g}} \operatorname{vol}_{\Sigma_{g}}=4 \pi(g-1)=-2 \pi \chi\left(\Sigma_{g}\right),
$$

with $\chi\left(\Sigma_{g}\right)$ the Euler number of $\Sigma_{g}$. This identifies the $\mathbb{R}^{2}$ bundle over $\Sigma_{g}$, with unit circle in $\mathbb{R}^{2}=\mathbb{C}$ having coordinate $\psi$, as $T^{*} \Sigma_{g}$. This is a local Calabi-Yau two-fold, with the $d=11$ solution describing the near-horizon limit of a stack of M5-branes wrapped on the zero-section $\Sigma_{g}$. The M5-branes have corresponding normal space $\mathbb{R}^{5}=\mathbb{C} \oplus \mathbb{R}^{3}$, and denoting the first factor by the complex line bundle $\mathcal{N}_{1}$, we see that

$$
\mathcal{N}_{1}=T^{*} \Sigma_{g} \otimes L_{3}
$$

where $A^{(3)}$ is a connection on a complex line bundle $L_{3}$ over the $d=5$ spacetime. For our solution with $Q_{1}=Q_{2}$ in $(2.39)$, this is a line bundle over the spindle $\Sigma=\mathbb{W} \mathbb{C P}_{\left[n_{-}, n_{+}\right]}^{1}$ with charge $Q_{3}$.

Finally, $\mu_{i}, i=1,2,3$, are constrained coordinates describing a round $S^{2} \subset \mathbb{R}^{3}$, with $\sum_{i=1}^{3} \mu_{i}^{2}=1$, and the connection terms are given by

$$
D \mu_{1}=\mathrm{d} \mu_{1}+2 A^{(1)} \mu_{2}, \quad D \mu_{2}=\mathrm{d} \mu_{2}-2 A^{(1)} \mu_{1}, \quad D \mu_{3}=\mathrm{d} \mu_{3} .
$$

Writing $\mu_{1}=\sin \vartheta \cos \nu, \mu_{2}=\sin \vartheta \sin \nu, \mu_{3}=\cos \vartheta$ in spherical polar coordinates, the twisted metric on $S^{2}$ is

$$
D \mu_{i} D \mu_{i}=\mathrm{d} \vartheta^{2}+\sin ^{2} \vartheta\left(\mathrm{d} \nu-2 A^{(1)}\right)^{2} .
$$

\footnotetext{
${ }^{3}$ We note that in [9] the uplifting formula is stated for $d=5$ gauge coupling set to $m_{\text {there }}=\frac{1}{2}$, while our $d=5$ solutions have this quantity set to 1 . We thus need to rescale our fields, and in the notation of [9] correspondingly $A_{\text {there }}^{3}=2 \sqrt{2} A^{(1)}, B_{\text {there }}=2 A^{(3)}$.
} 
Since $\nu$ has $\Delta \nu=2 \pi$, we see that writing $\mathbb{R}^{3}=\mathbb{C} \oplus \mathbb{R}$, the complex line $\mathbb{C}$ is twisted via $L_{1}^{2}$, with connection $2 A^{(1)}$, so we have

$$
\mathcal{N}_{2}=L_{1}^{2}
$$

The total normal bundle of the M5-branes is then $\mathcal{N}_{1} \oplus \mathcal{N}_{2} \oplus \mathbb{R}$, with $\mathbb{R}$ the $z$-axis direction in $\mathbb{R}^{3}$.

The M-theory four-form $G_{4}$ may also be read off from the expression in [9], although we won't need its explicit form in what follows.

\subsection{Central charge}

We begin by writing the metric (3.2) as

$$
\mathrm{d} s_{11}^{2}=(16 \Omega H(x))^{1 / 3}\left(\mathrm{~d} s_{\mathrm{AdS}_{3}}^{2}+\mathrm{d} s_{Y_{8}}^{2}\right)
$$

where we have uplifted the $d=5$ solution of section 2.4 with $Q_{1}=Q_{2}$. The eightdimensional metric on $Y_{8}$ is then

$$
\begin{aligned}
\mathrm{d} s_{Y_{8}}^{2}= & \frac{1}{4 P(x)} \mathrm{d} x^{2}+\frac{P(x)}{H(x)} \mathrm{d} \phi^{2}+\frac{X}{2 H(x)^{1 / 3}}\left(\mathrm{~d} \theta^{2}+\mathrm{d} s_{\Sigma_{g}}^{2}\right) \\
& +\frac{X}{2 \Omega H(x)^{1 / 3}} \sin ^{2} \theta\left(\mathrm{d} \psi+V+A^{(3)}\right)^{2}+\frac{1}{4 X^{2} \Omega H(x)^{1 / 3}} \cos ^{2} \theta D \mu_{i} D \mu_{i} .
\end{aligned}
$$

The central charge of the dual $d=2,(0,2)$ field theories is again given by formula $(2.24)$, where $G_{(3)}$ is computed via dimensional reduction on $Y_{8}$. We find

$$
\frac{1}{G_{(3)}}=\frac{L^{8}}{G_{(11)}} \int_{Y_{8}}(16 \Omega H(x))^{3 / 2} \operatorname{vol}_{8}=\frac{16 \pi^{2} L^{8} \Delta \phi}{3 G_{(11)}}\left(x_{2}-x_{1}\right) \operatorname{Vol}\left(\Sigma_{g}\right),
$$

where in our conventions the eleven-dimensional Newton constant is

$$
G_{(11)}=\frac{(2 \pi)^{8} \ell_{p}^{9}}{16 \pi}
$$

The central charge is then given by

$$
c=\frac{L^{9} \Delta \phi}{2 \pi^{5} \ell_{p}^{9}}\left(x_{2}-x_{1}\right) \operatorname{Vol}\left(\Sigma_{g}\right) .
$$

We also need to impose flux quantization on the four-form in order to have a consistent M-theory solution. Integrating the expression for $G_{4}$ in [9] over a copy of the $S^{4}$ fibre we get the number of M5 branes

$$
N \equiv \frac{1}{\left(2 \pi \ell_{p}\right)^{3}} \int_{S^{4}} G_{4}=\frac{L^{3}}{\pi \ell_{p}^{3}} .
$$

Substituting for the length scale $L$ in (3.13), $\operatorname{Vol}\left(\Sigma_{g}\right)$ given by (3.4), and also for $x_{1}, x_{2}$ in (2.39) and $\Delta \phi$ in (2.31) with $p=q$, we find the final central charge

$$
c=\frac{4 q^{2}\left(n_{-}-n_{+}-2 q\right)}{n_{-} n_{+}\left[n_{-}\left(n_{+}+2 q\right)-q\left(2 n_{+}+3 q\right)\right]}(g-1) N^{3} .
$$

This is the central charge of a family of $d=2,(0,2)$ theories obtained by wrapping $N$ M5branes on $\Sigma_{g} \times \Sigma$, with $\Sigma=\mathbb{W} \mathbb{C P}_{\left[n_{-}, n_{+}\right]}^{1}$ a spindle, and the integer $q$ with $0<q<\frac{1}{2}\left(n_{-}-n_{+}\right)$ determines the twisting of the normal bundle of the M5-branes. We shall recover this from a dual theory calculation in section 4 . 


\section{Field theory}

Although our $\mathrm{AdS}_{3} \times Y_{7}$ solutions were first constructed in [6], the description in the latter reference meant that the dual $d=2,(0,2)$ SCFTs were left unidentified. However, as in [3] our alternative $d=5$ construction of the solutions leads to a natural conjecture. Specifically, one begins with $\mathcal{N}=4 \mathrm{SYM}$ theory, which is holographically dual to the $\mathrm{AdS}_{5} \times S^{5}$ vacuum of the $d=5, \mathrm{U}(1)^{3}$ gauged supergravity theory. One then compactifies this theory on $\Sigma=\mathbb{W} \mathbb{C P}_{\left[n_{-}, n_{+}\right]}^{1}$, together with background fluxes for the $\mathrm{U}(1)^{3}$ Abelian symmetry given by (2.15). Recall here that $p_{1}=q, p_{2}=p, p_{3}=n_{-}-n_{+}-q-p$. The solution we have described suggests that this compactification of $\mathcal{N}=4 \mathrm{SYM}$ flows to a $d=2,(0,2)$ SCFT in the IR. We give evidence for this in sections 4.1 and 4.2 by computing the central charge and superconformal U(1) R-symmetry using purely field theory methods, finding precise agreement with the supergravity results. There is a similar interpretation of the M5-brane solutions in section 3. In section 4.3 we likewise compactify the theory on $N$ M5-branes on $\Sigma_{g} \times \Sigma$, with appropriate background fluxes, and compute the central charge of the $d=2,(0,2)$ SCFTs, again finding agreement with supergravity.

\subsection{D3-brane anomaly polynomial}

Generalizing the approach of [3], our starting point is the anomaly polynomial for $\mathcal{N}=$ 4 SYM, with background gauge field fluxes $F^{(I)}$ for the $\mathrm{U}(1)^{3} \subset \mathrm{SO}(6)$ Abelian global symmetry group of this theory. In the large $N$ limit this anomaly polynomial reads (see, for example, [17])

$$
\mathcal{A}_{4 \mathrm{~d}}=c_{1}\left(F^{(1)}\right) c_{1}\left(F^{(2)}\right) c_{1}\left(F^{(3)}\right) \frac{N^{2}}{2} .
$$

Here $c_{1}\left(F^{(I)}\right)$ denote the first Chern classes of the $\mathrm{U}(1)$ bundles with gauge field curvatures $F^{(I)}$, respectively.

We want to compactify this theory on $\Sigma=\mathbb{W} \mathbb{P}_{\left[n_{-}, n_{+}\right]}^{1}$, with fluxes given by $(2.15)$, and compute the anomaly polynomial of the resulting $d=2$ theory. For this we also need to take into account the $U(1)_{\mathcal{J}}$ global symmetry in $d=2$, coming from the $U(1)_{\mathcal{J}}$ isometry of $\Sigma$. Geometrically, this means that we want to compute the anomaly polynomial (4.1), where the six-manifold $Z_{6}$ on which it is defined is the total space of a $\Sigma$ fibration over $Z_{4}$. We may achieve this by introducing a corresponding connection form $\mathcal{A}_{\mathcal{J}}$ for $U(1)_{\mathcal{J}}$, and replacing $\mathrm{d} \varphi \rightarrow \mathrm{d} \varphi+\mathcal{A}_{\mathcal{J}}$. In doing so it is important that the $d=5$ Killing spinor is independent of $\tilde{\varphi}=\varphi$, which is true in the tilded gauge defined by (2.33), (2.35). We thus introduce the connection one-forms on $Z_{6}$ :

$$
\mathscr{A}^{(I)} \equiv\left(\frac{x-\alpha}{x+3 K_{I}}-\frac{2}{3}\right) \frac{\Delta \phi}{2 \pi}\left(\mathrm{d} \varphi+\mathcal{A}_{\mathcal{J}}\right) \equiv \rho_{I}(x)\left(\mathrm{d} \varphi+\mathcal{A}_{\mathcal{J}}\right), \quad I=1,2,3,
$$

which by construction restricts to the supergravity gauge field $\tilde{A}^{(I)}$ on each $\Sigma$ fibre. We then compute the curvature

$$
\mathscr{F}^{(I)} \equiv \mathrm{d} \mathscr{A}^{(I)}=\rho_{I}^{\prime}(x) \mathrm{d} x \wedge\left(\mathrm{d} \varphi+\mathcal{A}_{\mathcal{J}}\right)+\rho_{I}(x) \mathcal{F}_{\mathcal{J}},
$$

where $\mathcal{F}_{\mathcal{J}}=\mathrm{d} \mathcal{A}_{\mathcal{J}}$. By construction the integral of $\mathscr{F}^{(I)}$ over a fibre $\Sigma$ gives the flux $Q_{I}$, as in (2.14). The curvature form $\mathscr{F}^{(I)}$ defines a $\mathrm{U}(1)$ bundle $\mathcal{L}_{I}$ over $Z_{6}$ by taking 
$c_{1}\left(\mathcal{L}_{I}\right)=\left[\mathscr{F}^{(I)} / 2 \pi\right] \in H^{2}\left(Z_{6}, \mathbb{R}\right)$. We also define $c_{1}(\mathcal{J})=\left[\mathcal{F}_{\mathcal{J}} / 2 \pi\right] \in H^{2}\left(Z_{4}, \mathbb{Z}\right)$. In the anomaly polynomial we then write

$$
c_{1}\left(F^{(I)}\right)=\Delta_{I} c_{1}\left(R_{2 \mathrm{~d}}\right)+c_{1}\left(\mathcal{L}_{I}\right)
$$

where $R_{2 \mathrm{~d}}$ is the pull-back of a $\mathrm{U}(1)$ bundle over $Z_{4}$. The trial R-charges $\Delta_{I}$ satisfy the constraint $\Delta_{1}+\Delta_{2}+\Delta_{3}=2$, which is equivalent to the superpotential (in $\mathcal{N}=1$ language) having R-charge 2 .

The $d=2$ anomaly polynomial is then obtained by integrating $\mathcal{A}_{4 \mathrm{~d}}$ in (4.1) over $\Sigma$ :

$$
\mathcal{A}_{2 \mathrm{~d}}=\int_{\Sigma} \mathcal{A}_{4 \mathrm{~d}}=\frac{N^{2}}{2} \int_{\Sigma} c_{1}\left(F^{(1)}\right) c_{1}\left(F^{(2)}\right) c_{1}\left(F^{(3)}\right)
$$

which reads

$$
\begin{aligned}
\mathcal{A}_{2 \mathrm{~d}}=\frac{N^{2}}{2} \int_{\Sigma}\{ & \Delta_{1} \Delta_{2} \Delta_{3} c_{1}\left(R_{2 \mathrm{~d}}\right)^{3}+c_{1}\left(R_{2 \mathrm{~d}}\right)^{2}\left[\Delta_{2} \Delta_{3} c_{1}\left(\mathcal{L}_{1}\right)+\Delta_{1} \Delta_{3} c_{1}\left(\mathcal{L}_{2}\right)\right. \\
& \left.+\Delta_{1} \Delta_{2} c_{1}\left(\mathcal{L}_{3}\right)\right]+c_{1}\left(R_{2 \mathrm{~d}}\right)\left[\Delta_{3} c_{1}\left(\mathcal{L}_{1}\right) c_{1}\left(\mathcal{L}_{2}\right)+\Delta_{2} c_{1}\left(\mathcal{L}_{1}\right) c_{1}\left(\mathcal{L}_{3}\right)\right. \\
& \left.\left.+\Delta_{1} c_{1}\left(\mathcal{L}_{2}\right) c_{1}\left(\mathcal{L}_{3}\right)\right]+c_{1}\left(\mathcal{L}_{1}\right) c_{1}\left(\mathcal{L}_{2}\right) c_{1}\left(\mathcal{L}_{3}\right)\right\} .
\end{aligned}
$$

After a computation using the explicit functions $\rho_{I}(x)$ defined in (4.2), we find the integral in (4.6) gives

$$
\begin{aligned}
\mathcal{A}_{2 \mathrm{~d}}=\frac{N^{2}}{2}\{ & {\left[\frac{q \Delta_{2} \Delta_{3}}{n_{-} n_{+}}+\frac{p \Delta_{1} \Delta_{3}}{n_{-} n_{+}}+\frac{n_{-}-n_{+}-p-q}{n_{-} n_{+}} \Delta_{1} \Delta_{2}\right] c_{1}\left(R_{2 \mathrm{~d}}\right)^{2} } \\
& +\frac{\Delta_{1} f_{1}\left(n_{-}, n_{+}, p, q\right)+\Delta_{2} f_{2}\left(n_{-}, n_{+}, p, q\right)+\Delta_{3} f_{3}\left(n_{-}, n_{+}, p, q\right)}{3 n_{-}^{2} n_{+}^{2}\left(n_{-}+n_{+}\right)} c_{1}\left(R_{2 \mathrm{~d}}\right) c_{1}(\mathcal{J}) \\
& \left.+\frac{g_{1}\left(n_{-}, n_{+}, p, q\right) g_{2}\left(n_{-}, n_{+}, p, q\right)}{9 n_{-}^{3} n_{+}^{3}\left(n_{-}+n_{+}\right)^{2}} c_{1}(\mathcal{J})^{2}\right\}
\end{aligned}
$$

where we have defined

$$
\begin{aligned}
f_{1}\left(n_{-}, n_{+}, p, q\right) \equiv & -2\left(n_{-}^{2}+n_{+}^{2}\right)(p+q)+\left(n_{-}-n_{+}\right)\left(2 p^{2}+7 p q+4 q^{2}\right) \\
& +2 n_{-} n_{+}\left(-n_{-}+n_{+}+2 p+3 q\right)-q\left(5 p^{2}+5 p q+2 q^{2}\right), \\
f_{2}\left(n_{-}, n_{+}, p, q\right) \equiv & -2\left(n_{-}^{2}+n_{+}^{2}\right)(p+q)+\left(n_{-}-n_{+}\right)\left(2 q^{2}+7 p q+4 p^{2}\right) \\
& +2 n_{-} n_{+}\left(-n_{-}+n_{+}+2 q+3 p\right)-p\left(5 q^{2}+5 p q+2 p^{2}\right), \\
f_{3}\left(n_{-}, n_{+}, p, q\right) \equiv & -\left(n_{-}-n_{+}\right)\left(2 p^{2}+p q+2 q^{2}\right)-2 n_{-} n_{+}(p+q) \\
& +\left(2 p^{2}-p q+2 q^{2}\right)(p+q), \\
g_{1}\left(n_{-}, n_{+}, p, q\right) \equiv & -\left(n_{-}-n_{+}\right)\left(4 p^{2}+13 p q+4 q^{2}\right)+4\left(n_{-}-n_{+}\right)^{2}(p+q) \\
& +4 n_{-} n_{+}\left(n_{-}-n_{+}\right)+9 p q(p+q), \\
g_{2}\left(n_{-}, n_{+}, p, q\right) \equiv & \left(n_{-}-n_{+}\right)(p+q)+n_{-} n_{+}-\left(p^{2}+p q+q^{2}\right) .
\end{aligned}
$$




\section{$4.2 \quad c$-extremization and superconformal R-symmetry}

The coefficient of $\frac{1}{2} c_{1}\left(L_{i}\right) c_{1}\left(L_{j}\right)$ in the anomaly polynomial $\mathcal{A}_{2 \mathrm{~d}}$ is precisely $\operatorname{Tr} \gamma^{3} \mathcal{Q}_{i} \mathcal{Q}_{j}$, where the global symmetry $\mathcal{Q}_{i}$ is associated to the $\mathrm{U}(1)$ bundle $L_{i}$ over $Z_{4}$, and $\gamma^{3}$ is the $d=2$ chirality operator. We know from $c$-extremization [7] that the $d=2$ superconformal $\mathrm{U}(1)$ R-symmetry extremizes the trial function

$$
c_{\text {trial }}=3 \operatorname{Tr} \gamma^{3} R_{\text {trial }}^{2}
$$

over the space of possible R-symmetries. We accordingly set

$$
R_{\text {trial }}=R_{2 \mathrm{~d}}+\epsilon \mathcal{J},
$$

so that

$$
c_{\text {trial }}=3\left(\operatorname{Tr} \gamma^{3} R_{2 \mathrm{~d}}^{2}+2 \epsilon \operatorname{Tr} \gamma^{3} R_{2 \mathrm{~d}} \mathcal{J}+\epsilon^{2} \operatorname{Tr} \gamma^{3} \mathcal{J}^{2}\right) .
$$

Next we can substitute for the traces using (4.7), and extremize over the trial R-charges $\epsilon, \Delta_{I}$, subject to the constraint $\Delta_{1}+\Delta_{2}+\Delta_{3}=2$. We find the extremal values of these parameters to be

$$
\epsilon_{*}=\frac{n_{-} n_{+}\left(n_{-}+n_{+}\right)}{\left(n_{-}-n_{+}\right)(p+q)+n_{-} n_{+}-p^{2}-p q-q^{2}}, \quad \Delta_{1}^{*}=\Delta_{2}^{*}=\Delta_{3}^{*}=\frac{2}{3} .
$$

The right-moving central charge, which is equal to the central charge $c$ in the large $N$ limit, is then given by evaluating (4.9) on the superconformal R-symmetry with $\epsilon=\epsilon_{*}$ and $\Delta_{I}=\Delta_{I}^{*}$. We find

$$
c=\frac{3 p q\left(n_{-}-n_{+}-p-q\right)}{n_{-} n_{+}\left[\left(n_{-}-n_{+}\right)(p+q)+n_{-} n_{+}-p^{2}-p q-q^{2}\right]} N^{2} .
$$

This precisely matches the gravity computation (2.30), and moreover the R-symmetry (4.10) with $\epsilon=\epsilon_{*}$ and $\Delta_{I}=\Delta_{I}^{*}$ precisely matches $(2.38)$ !

\subsection{M5-brane anomaly polynomial}

The large $N$ limit of anomaly polynomial for $N$ M5-branes is given by (see e.g. [17])

$$
\mathcal{A}_{6 \mathrm{~d}}=\frac{1}{24} p_{2}(R) N^{3} .
$$

Here $R$ denotes the $\mathrm{SO}(5)_{R}$ symmetry of the M5-brane theory, which geometrically is identified with the normal bundle to the M5-brane in spacetime, and $p_{2}$ denotes the second Pontryagin class. The anomaly polynomial (4.14) is only valid to leading order in the large $N$ limit, and more generally receives $O(N)$ corrections involving also Pontryagin classes of the tangent bundle of the M5-brane.

The supergravity solution in section 3 has normal bundle twisted via the Cartan $\mathrm{U}(1) \times$ $\mathrm{U}(1) \subset \mathrm{SO}(5)_{R}$. Denoting these as two complex line bundles $\mathcal{N}_{1}, \mathcal{N}_{2}$, as in section 3.1, we may write the Pontryagin class in terms of Chern classes so that

$$
\mathcal{A}_{6 \mathrm{~d}}=\frac{1}{24} c_{1}\left(\mathcal{N}_{1}\right)^{2} c_{1}\left(\mathcal{N}_{2}\right)^{2} N^{3} .
$$


As described in section 3.1, the M5-branes are wrapped on $\Sigma_{g} \times \Sigma$, with $\Sigma=\mathbb{W} \mathbb{C P}_{\left[n_{-}, n_{+}\right]}^{1}$ a spindle. From the metric we saw that the normal bundles are

$$
\mathcal{N}_{1}=T^{*} \Sigma_{g} \otimes L_{3}, \quad \mathcal{N}_{2}=L_{1}^{2},
$$

where $L_{1} \cong L_{2}$ because we have set the first two charges equal, $Q_{1}=Q_{2}$, and $T^{*} \Sigma_{g}$ is the cotangent bundle to the Riemann surface $\Sigma_{g}$, with total space being a Calabi-Yau two-fold. The corresponding first Chern class gives minus the Euler number of this Riemann surface:

$$
\int_{\Sigma_{g}} c_{1}\left(T^{*} \Sigma_{g}\right)=2(g-1) .
$$

Since $c_{1}\left(\mathcal{N}_{1}\right)=c_{1}\left(T^{*} \Sigma_{g}\right)+c_{1}\left(F^{(3)}\right)$, we may first integrate the anomaly polynomial (4.15) over $\Sigma_{g}$ to obtain

$$
\begin{aligned}
\mathcal{A}_{4 \mathrm{~d}} & =\int_{\Sigma_{g}} \mathcal{A}_{6 \mathrm{~d}}=\frac{N^{3}}{24} \cdot 2\left(\int_{\Sigma_{g}} c_{1}\left(T^{*} \Sigma_{g}\right)\right) c_{1}\left(F^{(3)}\right)\left[2 c_{1}\left(F^{(1)}\right)\right]^{2} \\
& =c_{1}\left(F^{(3)}\right) c_{1}\left(F^{(1)}\right)^{2} \cdot \frac{2}{3}(g-1) N^{3} .
\end{aligned}
$$

We have thus effectively reduced to the four-dimensional anomaly polynomial (4.1), where we must take $F^{(1)}=F^{(2)}$ and the overall factor of $N^{2} / 2$ should be replaced by $2(g-1) N^{3} / 3$. The rest of the computation of the $d=2$ anomaly polynomial, obtained by integrating $\mathcal{A}_{4 \mathrm{~d}}$ over the spindle, and extracting the central charge, then goes through mutatis mutandis. The final central charge is thus given by (4.13) with $p=q$, and replacing an overall factor of $N^{2} / 2$ by $2(g-1) N^{3} / 3$. We obtain

$$
c=\frac{4\left(n_{-}-n_{+}-2 q\right) q^{2}}{n_{-} n_{+}\left[n_{-}\left(n_{+}+2 q\right)-q\left(2 n_{+}+3 q\right)\right]}(g-1) N^{3} .
$$

This perfectly matches the supergravity result (3.15).

\section{Discussion}

In this paper we have constructed a five-parameter family of $\mathrm{AdS}_{3} \times Y_{7}$ solutions of type IIB supergravity, in which $Y_{7}$ is the total space of an $S^{5}$ fibration over a spindle $\Sigma=$ $\mathbb{W} \mathbb{C P}_{\left[n_{-}, n_{+}\right]}^{1}$. In addition to the coprime positive integers $n_{-}>n_{+}$specifying $\Sigma$, the solution is also characterized by positive integers $q, p$ that determine the twisting of the $S^{5}$ over $\Sigma$, together with the quantized five-form flux $N$ through the $S^{5}$. Setting $q=p=\frac{1}{3}\left(n_{-}-n_{+}\right)$ recovers the solutions of [3], and we have thus generalized those solutions via the addition of the twisting parameters $q, p$. As in [3] we interpret these as the near-horizon limit of $N$ D3-branes wrapping the spindle $\Sigma$, with $q$ and $p$ now determining the background $\mathrm{U}(1)^{3}$ Abelian R-symmetry fluxes via (1.3), (1.4). As a consistency check on this interpretation, we have reproduced the gravity formulas for the central charge and superconformal Rsymmetry from a dual field theory computation, starting with the anomaly polynomial of $\mathcal{N}=4 \mathrm{SYM}$ and performing an appropriate twisted compactification on $\Sigma$. Similarly, we 
have constructed a four-parameter family of $\mathrm{AdS}_{3} \times Y_{8}$ solutions of $d=11$ supergravity, which we interpret as the near-horizon limit of $N$ M5-branes wrapping $\Sigma_{g} \times \Sigma$, and we have reproduced the gravitational formula for the central charge from a dual field theory computation.

One of the most interesting features of the solutions of [3], that our solutions inherit, is that the Killing spinors on $\Sigma$ are not simply given by a topological twist. This is exemplified by the formula (1.5), where the right hand side is not the Euler number $\chi(\Sigma)$ given in (1.2). An analogous family of $d=4 \mathrm{AdS}_{2} \times \Sigma$ solutions was studied in more detail in [4]. In fact these are near-horizon limits of full $d=4$ accelerating extremal black hole solutions, where it is the acceleration parameter that effectively leads to the conical deficit singularities on the black hole horizon $\Sigma$. In this case we also have a UV geometry, on the conformal boundary of the black holes in $\mathrm{AdS}_{4}$, and remarkably one finds that there is a topological twist on the copy of $\Sigma$ in the UV, but that it has effectively been cut in half along an equator by the acceleration horizon of the black hole! The spinor is a different constant on the two halves of the spindle in the UV. The physical interpretation of this, in terms of wrapped branes, is still somewhat obscure, and it would be interesting to find an analogous class of black string solutions in $d=5, \mathrm{U}(1)^{3}$ gauged supergravity that have our solutions as a near-horizon limit. Alternatively, one could directly attempt to study $\mathcal{N}=4 \mathrm{SYM}$ on a rigid supersymmetric background $\mathbb{R}^{2} \times \Sigma$, with background fluxes (1.3), (1.4), making more explicit the twisting of the fields and their boundary conditions at the conical singularities of $\Sigma$. In particular, one might envisage that additional data needs to be specified at the conical singularities, in describing the behaviour of the fields. We note that the anomaly polynomial method gives the correct supergravity result, despite the presence of the conical deficit singularities on $\Sigma$, and it would be interesting to justify this more carefully in such an analysis.

Finally, in section 3 we uplifted the $d=5, Q_{1}=Q_{2}$ solutions on the Maldacena-Núnez solution, but we also commented that one can uplift on other internal six-manifolds $N_{6}$ to obtain solutions to $d=11$ supergravity. Such solutions [16] describe M5-branes wrapped on a Riemann surface, in general with punctures, which are then further wrapped on the spindle $\Sigma$ to obtain $d=2,(0,2)$ SCFTs. It would be interesting to investigate these solutions in more detail, in particular computing the central charge in gravity and via the anomaly polynomial for the theory on $N$ M5-branes. We leave this, together with other interesting generalizations, for future work.

\section{Acknowledgments}

We thank Dario Martelli for helpful discussions. The work of JFS was supported in part by STFC grant ST/T000864/1.

Open Access. This article is distributed under the terms of the Creative Commons Attribution License (CC-BY 4.0), which permits any use, distribution and reproduction in any medium, provided the original author(s) and source are credited. 


\section{References}

[1] J.M. Maldacena and C. Núñez, Supergravity description of field theories on curved manifolds and a no go theorem, Int. J. Mod. Phys. A 16 (2001) 822 [hep-th/0007018] [INSPIRE].

[2] E. Witten, Topological Quantum Field Theory, Commun. Math. Phys. 117 (1988) 353 [INSPIRE].

[3] P. Ferrero, J.P. Gauntlett, J.M. Pérez Ipiña, D. Martelli and J. Sparks, D3-branes Wrapped on a Spindle, Phys. Rev. Lett. 126 (2021) 111601 [arXiv:2011.10579] [InSPIRE].

[4] P. Ferrero, J.P. Gauntlett, J.M.P. Ipiña, D. Martelli and J. Sparks, Accelerating Black Holes and Spinning Spindles, arXiv:2012.08530 [INSPIRE].

[5] H.K. Kunduri and J. Lucietti, Near-horizon geometries of supersymmetric AdS $S_{5}$ black holes, JHEP 12 (2007) 015 [arXiv:0708.3695] [INSPIRE].

[6] J.P. Gauntlett, N. Kim and D. Waldram, Supersymmetric $A d S_{3}, A d S_{2}$ and Bubble Solutions, JHEP 04 (2007) 005 [hep-th/0612253] [INSPIRE].

[7] F. Benini and N. Bobev, Exact two-dimensional superconformal R-symmetry and c-extremization, Phys. Rev. Lett. 110 (2013) 061601 [arXiv:1211.4030] [INSPIRE].

[8] L.J. Romans, Gauged $N=4$ Supergravities in Five-dimensions and Their Magnetovac Backgrounds, Nucl. Phys. B 267 (1986) 433 [inSPIRE].

[9] J.P. Gauntlett and O. Varela, $D=5 \mathrm{SU}(2) x \mathrm{U}(1)$ Gauged Supergravity from $D=11$ Supergravity, JHEP 02 (2008) 083 [arXiv:0712.3560] [InSPIRE].

[10] S.M. Hosseini, K. Hristov and A. Zaffaroni, Rotating multi-charge spindles and their microstates, arXiv:2104.11249 [INSPIRE].

[11] M. Cvetič et al., Embedding AdS black holes in ten-dimensions and eleven-dimensions, Nucl. Phys. B 558 (1999) 96 [hep-th/9903214] [INSPIRE].

[12] J.D. Brown and M. Henneaux, Central Charges in the Canonical Realization of Asymptotic Symmetries: An Example from Three-Dimensional Gravity, Commun. Math. Phys. 104 (1986) 207 [INSPIRE].

[13] C. Couzens, J.P. Gauntlett, D. Martelli and J. Sparks, A geometric dual of c-extremization, JHEP 01 (2019) 212 [arXiv:1810.11026] [INSPIRE].

[14] J.P. Gauntlett and N. Kim, Geometries with Killing Spinors and Supersymmetric AdS Solutions, Commun. Math. Phys. 284 (2008) 897 [arXiv:0710.2590] [InSPIRE].

[15] H. Lü, C.N. Pope and T.A. Tran, Five-dimensional $N=4, \mathrm{SU}(2) \times \mathrm{U}(1)$ gauged supergravity from type IIB, Phys. Lett. B 475 (2000) 261 [hep-th/9909203] [INSPIRE].

[16] D. Gaiotto and J. Maldacena, The Gravity duals of $N=2$ superconformal field theories, JHEP 10 (2012) 189 [arXiv:0904.4466] [inSPIRE].

[17] S.M. Hosseini, K. Hristov, Y. Tachikawa and A. Zaffaroni, Anomalies, Black strings and the charged Cardy formula, JHEP 09 (2020) 167 [arXiv:2006.08629] [INSPIRE]. 